\title{
Optimized Technical and Economic Evaluation of the Connection Point of Distributed Generators
}

\author{
Milton Luiz Neri Pereira ${ }^{1}$ • Antonio Padilha-Feltrin ${ }^{2}$ - Augusto C. Rueda-Medina ${ }^{2}$
}

Received: 14 April 2015 / Revised: 23 June 2015 / Accepted: 15 July 2015 / Published online: 6 August 2015

(C) Brazilian Society for Automatics-SBA 2015

\begin{abstract}
In most studies, the problem of allocation and sizing of the distributed generation is addressed considering that the distributed generators (DGs) could be installed in any bus of the system. Thus, these studies bring the optimal or best allocation place for the DGs without considering that in a real implementation not all buses are valid candidates due to restrictions in the system, such as environmental, social, and geographical aspects, for example. In this sense, the problem treated in this work consists of evaluating the allocating point of DGs in distribution systems based on technical and economic aspects, assuming that they are not necessarily located near the optimal or best allocation bus of the system. Two interests are investigated: on one hand, the problem 1, formulated as a multi-objective optimization (MO) problem and defined from the point of view of the independent energy producer (IEP) which owns the DGs; and, on the other hand, the problem 2, also formulated as a MO problem, but defined from the point of view of the distribution system operator (DSO). The results for the two problems were a set of solutions that established a conflict between the IEP and the DSO because the alternative that proved the most promising for the IEP was the less attractive for the DSO. The set of solutions found through the used solution technique constitutes a flexible environment that allows the IEP and the DSO to evaluate each proposed solution and decide on the one that is closest to the interests of both parties.
\end{abstract}

Keywords Distributed generation - Electric distribution system $\cdot$ Multi-objective optimization

$凶$ Augusto C. Rueda-Medina

aucerume@gmail.com

1 Universidade do Estado de Mato Grosso, Sinop, Brazil

2 Universidade Estadual Paulista, Ilha Solteira, Brazil

\section{List of Symbols}

$\tau$

$\mathrm{TP}_{\mathrm{DG}}^{(\tau)}$

$f_{\mathrm{A} i}$

$P_{\mathrm{DG} i}^{(\tau)}, Q_{\mathrm{DG} i}^{(\tau)}$

$n_{\text {DG }}$

$\mathrm{TC}_{\text {Inst }}^{(\tau)}$

$\mathrm{TC}_{\mathrm{Inv}}^{(\tau)}$

$c_{\text {Inv } i}^{(\tau)}$

$\mathrm{TC}_{\mathrm{Op} / \mathrm{Man}}^{(\tau)}$

$c_{\mathrm{Op} / \mathrm{Man}_{i}}^{(\tau)}$

$\varphi_{i}^{(\tau)}$

$\alpha$

$T$

$\mathrm{TC}_{\text {Line }}^{(\tau)}$

$c^{(\tau)}{ }^{\text {Line }}$

$c_{\text {Line } i, j}$

$D_{i, j}$

$n_{\mathrm{B}}, n_{\mathrm{Br}}$

$\mathrm{TC}_{\text {Char }}^{(\tau)}$

$c_{\mathrm{Char} i, j}^{(\tau)}$

$P_{\mathrm{Cal} i}^{(\tau)}, Q_{\mathrm{Cal} i}^{(\tau)}$

$P_{\mathrm{Spe}_{i}}^{(\tau)}, Q_{\mathrm{Spe}_{i}}^{(\tau)}$

$V_{\operatorname{Min} i}, V_{\operatorname{Max} i}$

$P_{\operatorname{Min} i}^{(\tau)}, Q_{\operatorname{Min} i}^{(\tau)}$

$P_{\operatorname{Max} i}^{(\tau)}, Q_{\operatorname{Max} i}^{(\tau)}$
An allocation scenario

Total active power generated by the DGs Availability factor of DG $i$

Active and reactive power generated by DG $i$

Number of DGs

Total installation cost of DGs

Total investment cost for DGs

Initial investment cost for DG $i$

Total operation and maintenance cost of DGs

Operation and maintenance cost for DG $i$

Present value of cost of DG $i$

Interest rate

Study period

Total connection line cost for DGs

Connection line cost for DG $i$ in bus $j$

Distance from DG $i$ to connection point $j$

Number of system buses and branches

Total cost due to the use of the EDS by DG $i$ in bus $j$

Cost due to the use of the EDS by DG $i$ in bus $j$

Active and reactive power calculated in bus $i$

Active and reactive power specified in bus $i$ Minimum and maximum voltages in bus $i$

Minimum active and reactive power limits of DG $i$

Maximum active and reactive power limits of DG $i$ 


\begin{tabular}{|c|c|}
\hline$p f_{\mathrm{DG} i}^{(\tau)}$ & Power factor of DG $i$ \\
\hline$E_{\mathrm{Loss}}^{(\tau)}, E_{\mathrm{Loss}}^{\mathrm{DG}(\tau)}$ & Energy losses without and with DGs \\
\hline & Number of load levels \\
\hline$V_{i}^{(\tau)}$ & Voltage in bus $i$ \\
\hline$Z_{i}, R_{i}$ & Impedance and resistance of branch $i$ \\
\hline$\Delta t_{i}$ & $\begin{array}{l}\text { Number of hours per day with load in level } \\
i\end{array}$ \\
\hline$V_{\mathrm{B}_{i, j}}^{(\tau)}, V_{\mathrm{B}, \mathrm{DG}_{i, j}}^{(\tau)}$ & $\begin{array}{l}\text { Voltages in branch } i \text { with load in level } j \\
\text { before and after installing the DGs }\end{array}$ \\
\hline $\operatorname{VEL}^{(\tau)}$ & Valuation of energy losses \\
\hline $\mathrm{EC}_{i}^{(\tau)}$ & Energy cost in load level $i$ \\
\hline & Number of sections of the system \\
\hline $\mathrm{VNE}_{\mathrm{DG} i, j}^{(\tau)}$ & $\begin{array}{l}\text { Valuation of energy not supplied by the EDS } \\
\text { but restored by the DGs in load level } i \text { in } \\
\text { section } j\end{array}$ \\
\hline $\operatorname{SEC}_{\mathrm{DG} i, j}^{(\tau)}$ & $\begin{array}{l}\text { Supplied energy cost restored by the DGs in } \\
\text { load level } i \text { in section } j\end{array}$ \\
\hline $\mathrm{RL}_{\mathrm{DG} i}^{(\tau)}$ & Load restored by the DGs in section $i$ \\
\hline$A C^{(\tau)}$ & System adequacy costs \\
\hline $\operatorname{PFC}^{(\tau)}$ & Protection fixed costs \\
\hline$n_{\text {In }}$ & Number of load intervals \\
\hline & Number of protection devices \\
\hline $\mathrm{AQC}_{i, j}^{(\tau)}$ & $\begin{array}{l}\text { Acquisition costs of protection device } i \\
\text { operating in interval } j\end{array}$ \\
\hline & Protection service costs \\
\hline $\operatorname{In}_{i, j}^{(\tau)}, U n_{i, j}^{(\tau)}$ & $\begin{array}{l}\text { Installation and uninstallation costs of pro- } \\
\text { tection device } i \text { in interval } j\end{array}$ \\
\hline $\mathrm{MC}_{i, j}^{(\tau)}$ & $\begin{array}{l}\text { Maintenance costs of protection device } i \\
\text { operating in interval } j\end{array}$ \\
\hline $\operatorname{PMC}^{(\tau)}$ & Protection maintenance costs \\
\hline$f i t_{i, j}, o f_{i, j}$ & $\begin{array}{l}\text { Generic fitness and objective functions val- } \\
\text { ues of individual } j \text { in generation } i \text { of the MO } \\
\text { algorithm }\end{array}$ \\
\hline$n_{\mathrm{V} i}$ & $\begin{array}{l}\text { Number of violated constraints in generation } \\
i \text { of the MO algorithm }\end{array}$ \\
\hline$n_{\mathrm{G}}$ & $\begin{array}{l}\text { Total number of generations of the MO algo- } \\
\text { rithm }\end{array}$ \\
\hline$n_{\mathrm{N}}, n_{\mathrm{M}}$ & $\begin{array}{l}\text { Size of the external and population sets of } \\
\text { the MO algorithm }\end{array}$ \\
\hline$\beta_{i, j}$ & $\begin{array}{l}\text { Dimensional adjustment factor of individual } \\
j \text { in generation } i \text { associated with the generic } \\
\text { objective function } o f_{i, j}\end{array}$ \\
\hline$\omega_{i, j, k}, \Omega_{i, j, k}$ & $\begin{array}{l}\text { Penalty coefficient and function associated } \\
\text { with the violated constraint } k \text { of individual } \\
j \text { in generation } i \text { of the } \mathrm{MO} \text { algorithm }\end{array}$ \\
\hline$\underline{u}_{g, n, i}, \bar{u}_{g, n, i}$ & $\begin{array}{l}\text { Minimum and maximum limits of constraint } \\
k \text { of individual } j \text { in generation } i \text { of the MO } \\
\text { algorithm }\end{array}$ \\
\hline$u_{i, j, k}$ & $\begin{array}{l}\text { Actual value of the violated constraint } k \text { of } \\
\text { individual } j \text { in generation } i \text { of the MO algo- } \\
\text { rithm }\end{array}$ \\
\hline
\end{tabular}

\section{Introduction}

Several factors have contributed restructuring the electricity sector, among them: population growth, increase in demand for electricity, development of new technologies and need for more cost-efficient and cleaner electrical systems.

This process occurred in many countries and has significantly contributed to the elimination of legal and economic barriers, rising new electricity generating agents. As a result, there is an increase in decentralized generation through renewable energy sources.

An immediate consequence of this scenario is the increase in the number of requests from independent energy producers (IEPs), together with distribution systems operators (DSOs), of authorization to connect generators directly into the electric distribution systems (EDSs), coining the term distributed generation.

In most countries, distributed or decentralized generation is understood as the energy generated from an unit located on the area of a DSO and close to the consumption point. In Brazil, the legislation refers to distributed generation as the electricity contracted from public call and generated exclusively for the DSO to attend up to $10 \%$ of its own demand. According to the Brazilian Electricity Regulatory Agency (ANEEL, from Agência Nacional de Energia Elétrica), the DSOs are not prevented from owning distributed generators (DGs), and it is guaranteed to consumers the possibility of requesting the connection of their own generators to the EDS (ANEEL 2005).

The DG's impacts on the EDS have been widely discussed in several works already recognized in the literature Barker and Demello (2000), Brown and Freeman (2001), Nazari and Parniani (2006), Nuroglu and Arsoy (2008), Hsieh (2011). It is known that, from a technical point of view, the introduction of DGs in networks traditionally designed for supplying only loads could have both beneficial as harmful effects, which depends on the relationship of various factors. Thus, the study of the EDSs in this context becomes a highly complex task. Even the traditional process of planning the expansion has high complexity due to features such as conflict of objectives, a large number of variables, dynamic nature of the problem, uncertainties and high investments involved. These challenges not only remain, but they are enhanced in the presence of the DGs (Neimane 2001).

In the study of EDS with DGs, several criteria should be considered, for example: (1) security; (2) charge for normal or contingency operation; (3) voltage for normal or contingency operation; (4) product and service quality; (5) reliability; (6) economic viability; and (7) environmental sustainability.

Consumers located in the DSO concession area could be considered as potential energy producers. In this likely sce- 
nario, the performance of these investors lends itself to the generation and sale of electricity with the DSO. Therefore, it is of interest to develop methodologies and tools for analysis of the energy to be generated, location and design of the DGs, reliability, energy losses, and economic considerations.

The problem of allocation and sizing of the distributed generation has already been addressed by several researchers. They have employed conventional optimization techniques, such as generalized reduced gradient (Rau and Wan 1994) and Newton-Raphson power flows (Ghosh et al. 2010); commercial software, which is composed of linear and nonlinear solver tools (Keane and O'Malley 2005; Atwa and El-Saadany 2011); analytical approaches using sensitivity factors, based on multi-objective indexes (Ochoa et al. 2008), equivalent current injection techniques (Gözel and Hocauglu 2009), and deriving generalized formulas that estimate the amount of reduction in active power losses (Mahmoud et al. 2015); and combinatorial optimization techniques based on artificial intelligence, such as evolutionary algorithms Abou El-Ela et al. (2010), Ganguly and Samajpati (2015), and particle swarm Wen et al. (2015). The major objectives considered in these studies are to minimize system losses (Rau and Wan 1994; Ghosh et al. 2010; Ochoa et al. 2008; Gözel and Hocauglu 2009; Mahmoud et al. 2015; Abou El-Ela et al. 2010; Ganguly and Samajpati 2015, and to minimize investment and operation costs (Keane and O'Malley 2005; Atwa and El-Saadany 2011; Wen et al. 2015). However, further objectives have also been addressed, such as to minimize line loadings (Rau and Wan 1994; Abou El-Ela et al. 2010), and to maximize the DGs capacity with respect to the technical constraints Keane and O'Malley (2005). A review on optimal sizing and siting of distributed generation could be found in Gupta et al. (2014).

In all aforementioned studies, the problem of allocation and sizing of the distributed generation is addressed considering that the DGs could be installed in any bus of the system. Thus, these studies bring the optimal or best allocation place for the DGs without considering that in a real implementation not all buses are valid candidates due to restrictions in the system, such as environmental, social, and geographical aspects. For example, the primary energy source could be away from the best candidate bus for allocation of the DG, or the best installation option might be in an area of great concentration of people. In this sense, the problem of DGs interconnection to the EDS is treated in this work assuming that the DGs are not necessarily located near to the optimal or best allocation bus of the system.

In this work, a proposal for studies of the EDS with the interconnection of DGs in the view of both perspectives (the IEPs and the DSO) is presented. A similar work was presented in Hsieh (2011), in which it is proposed a multi-objective optimization (MO) formulation considering expansion factors and characteristics and operating limita- tions of the EDS, as well as benefits of reducing energy losses. The bi-objective problem presented in Hsieh (2011) is solved by a compromise programing approach and particle swarm optimization to normalize and combine the two objectives into a single function and assign weights according to the importance of the objectives. Different weights and planning periods can cause different candidate solutions and the decision would be in accordance with the preference of the planner.

The objectives for the study presented in this paper are shown below:

- Determine the location and size of the DGs to be installed in the EDS, from the point of view of the IEPs (defined as problem 1) and the DSO (defined as problem 2);

- Explore a MO technique to find good-quality solutions for the allocation and size of the DGs, and the costs associated with their connection to the EDS, in the IEP's perspective.

- Find good-quality solutions in the DSO's perspective, considering the solutions found for the IEP's perspective.

- Find the possible connection alternatives of the DGs, from the perspective of both IEP and DSO.

The study starts by developing two mathematical models. In the first one, from the point of view of the IEP, a model is proposed with two objectives in order to maximize the active power to be generated by the DGs and minimize the cost associated with this generation. This cost relates to investment, operation and maintenance of the DGs, cost due to the construction of express lines to transport the energy produced by the DGs to the respective connection points in the EDS, and cost due to the use of the EDS by the DGs. In the second one, in the DSO's perspective, a three-objective model is proposed aiming to maximize the valuation of energy losses (difference between losses with and without DGs allocated in the system), maximize the reliability of the EDS, and minimize investment costs associated with protection devices installed in the EDS due to the connection of the DGs. To solve the problems proposed in this work, the strength Pareto evolutionary algorithm 2 (SPEA2) is used (Zitzler 1999).

The valuation of energy losses is obtained through a mathematical function that returns the difference between energy losses with and without DGs in the EDS. Reliability is measured by cost for energy not met by the EDS in response to actions of the protection system after the occurrence of a transient or permanent disturb, which was restored by the DGs. In this context, it is assumed that during the interruption of power supply to a region served by the EDS, and being this an isolated region, the DGs could operate in islanded mode through an appropriate protection scheme, provided they are 
not connected to the contingency region. On the other hand, the EDS adequacy costs are related to the acquisition, installation and maintenance of protection devices regarding the operation of the DGs in islanded mode.

The main contributions of this paper are summarized below:

- Evaluate the technical and economic feasibility of supply the energy required by the electrical system from DGs considering, on one hand, the IEPs and, on the other hand, the DSO.

- Provide solutions that could be analyzed and used as mediators in the discussion between the IEPs and the DSO in order to reach a consensus with respect to the connection point of the DGs. Provide analysis tools for decision-making and help the IEPs and the DSO to take a significant acting with positive impacts on the electrical system.

This paper is structured as follows: In Sect. 2, the mathematical formulation of the proposed problems is presented; in Sect. 3, the power flow and MO algorithm used for the solution of the formulated problems are described; in Sect. 4, the obtained results are presented and a discussion about them is carried out; and, finally, in Sect. 5, the conclusions are presented.

\section{Mathematical Formulation of the Proposed Problems}

As commented before, the proposal presented in this paper is composed of two problems from two points of view, the IEPs and the DSO, defined as problem 1 and problem 2, respectively. In this section, the mathematical formulation of these problems is presented.

\subsection{Mathematical Formulation of Problem 1}

The problem 1 is formulated as a MO problem, whose objective functions are to maximize the capacity of the DGs and minimize the costs involved due to the allocation of the DGs, subject to a set of constraints. The MO mathematical formulation of problem 1 is presented below.

$$
\begin{aligned}
\operatorname{Max} \mathrm{TP}_{\mathrm{DG}}^{(\tau)} & =\sum_{i=1}^{n_{\mathrm{DG}}} f_{\mathrm{A} i} P_{\mathrm{DG} i}^{(\tau)} \\
\operatorname{Min} \mathrm{TC}_{\text {Inst }}^{(\tau)} & =\mathrm{TC}_{\text {Inv }}^{(\tau)}+\mathrm{TC}_{\mathrm{Op} / \mathrm{Man}}^{(\tau)}+\mathrm{TC}_{\text {Line }}^{(\tau)} \\
& +\mathrm{TC}_{\mathrm{Char}}^{(\tau)}
\end{aligned}
$$

Subject to:

$$
\begin{array}{lr}
P_{\mathrm{Cal} i}^{(\tau)}-P_{\mathrm{Spe}_{i}}^{(\tau)}=0 & \forall i=1, \ldots, n_{\mathrm{B}} \\
Q_{\mathrm{Cal} i}^{(\tau)}-Q P_{\mathrm{Spe}_{i}}^{(\tau)}=0 & \forall i=1, \ldots, n_{\mathrm{B}} \\
V_{\mathrm{Min} i} \leq V_{i}^{(\tau)} \leq V_{\mathrm{Max} i} & \forall i=1, \ldots, n_{\mathrm{B}} \\
P_{\mathrm{Min} i}^{(\tau)} \leq P_{\mathrm{DG} i}^{(\tau)} \leq P_{\mathrm{Max} i}^{(\tau)} & \forall i=1, \ldots, n_{\mathrm{DG}} \\
Q_{\mathrm{Min} i}^{(\tau)} \leq Q_{\mathrm{DG} i}^{(\tau)} \leq Q_{\mathrm{Max} i}^{(\tau)} & \forall i=1, \ldots, n_{\mathrm{DG}} \\
P_{\mathrm{DG} i}^{(\tau)} \tan \left(\operatorname{acos}\left(p f_{\mathrm{DG} i}^{(\tau)}\right)\right) \leq Q_{\mathrm{DG} i}^{(\tau)} & \forall i=1, \ldots, n_{D G}
\end{array}
$$

The expression for the objective function which considers the active power generated and transmitted to the EDS by the DGs is presented in (1). The objective function called as allocation costs, presented in (2), was used to consider several costs components which are responsibility of the IEP due to the installation of the DGs; these costs are due to investment $\left(\mathrm{TC}_{I n v}\right)$, operation and maintenance $\left(\mathrm{TC}_{O P / \text { Man }}\right)$, building of the air connection line to the EDS $\left(\mathrm{TC}_{\text {Line }}\right)$ and connection charges paid to the DSO for the use of the EDS (TC Char), as presented in (9)-(12). Each DGs allocation scenario must meet the active and reactive power balance constraints at buses of the EDS, according to (3) and (4), respectively. The system voltages should be kept within limits set by standards established by the regulatory agency, according to constraint (5). The DGs should be within limits inherent to the size of their equipments to ensure its full operation in normal state, as guaranteed by constraints (6) and (7). In this study, the DGs are modeled as PQ-type, with the excitation system controlling a constant power factor; therefore, the relation between active and reactive power generated by the DGs is ensured through (8).

$$
\begin{aligned}
& \mathrm{TC}_{\mathrm{Inv}}^{(\tau)}=\sum_{i=1}^{n_{\mathrm{DG}}} c_{\mathrm{Inv} i}^{(\tau)} P_{\mathrm{DG} i}^{(\tau)} \\
& \mathrm{TC}_{\mathrm{Op} / \mathrm{Man}}^{(\tau)}=\sum_{i=1}^{n_{\mathrm{DG}}} f_{\mathrm{A} i} c_{\mathrm{Op} / \mathrm{Man}_{i}}^{(\tau)} P_{\mathrm{DG} i}^{(\tau)} \varphi_{i}^{(\tau)} \\
& \mathrm{TC}_{\mathrm{Line}}^{(\tau)}=\sum_{i=1}^{n_{\mathrm{DG}}} \sum_{j=1}^{n_{\mathrm{B}}} c_{\mathrm{Line} i, j}^{(\tau)} D_{i, j} \\
& \mathrm{TC}_{\mathrm{Char}}^{(\tau)}=12 \sum_{i=1}^{n_{\mathrm{DG}}} \sum_{j=1}^{n_{\mathrm{B}}} c_{\mathrm{Char} i, j}^{(\tau)} P_{\mathrm{DG} i}^{(\tau)} \varphi_{i}^{(\tau)}
\end{aligned}
$$

Several studies have been conducted to find the optimal location of DGs in medium-voltage (MV) networks; however, it is not generally considered that the DGs are unlikely to be physically located next to the point of connection to the EDS. The location of the DGs depends on the availability of viable space for their construction, availability of the primary energy source, environmental and social impacts, among 
others. Therefore, in most real cases, the best location places for the DGs are not close to the network, and it is required a MV line to connect the DGs to the EDS, which is an investment that must be in charge of the IEP. In this study, the expression given in (11) was used to represent the construction costs of the connection line between the DGs and the EDS.

In this study, operation, maintenance, and connection charges costs are spread over a year. Thus, the function of present value shown in (13) is used to convert future values associated with these costs in present values. It was also considered that the DGs can be connected at any load bus of the EDS.

$$
\varphi_{i}^{(\tau)}=\left(\frac{1}{(1+\alpha)^{T}}\right)
$$

\subsection{Mathematical Formulation of Problem 2}

The main objective in this part of the proposal is to find the costs associated with the EDS after allocation and operation of the DGs. In this sense, it is assumed that the DGs are connected along the feeder and the necessary adjustments will be in charge of the DSO. The MO mathematical formulation of problem 2 is presented below.

$$
\begin{aligned}
& \operatorname{Max} \operatorname{VEL}^{(\tau)}=365\left(E_{\text {Loss }}^{(\tau)}-E_{\mathrm{Loss}}^{\mathrm{DG}(\tau)}\right) \mathrm{EC}_{i}^{(\tau)} \varphi_{i}^{(\tau)}
\end{aligned}
$$

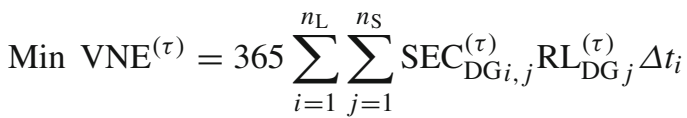

\section{Subject to: (3)-(8)}

The objective function proposed in (14) consists of the valuation of energy losses (VEL), which is the difference between losses without and with DGs brought to present values and assuming a year with 365 days. Losses without and with DGs are obtained according to (17) and (18), respectively.

$$
\begin{aligned}
& E_{\mathrm{Loss}}^{(\tau)}=\sum_{i=1}^{n_{\mathrm{Br}}} \sum_{j=1}^{n_{\mathrm{L}}} \frac{\left(V_{\mathrm{B}_{i, j}}^{(\tau)}\right)^{2}}{R_{i}} \Delta t_{j} \\
& E_{\mathrm{Loss}}^{\mathrm{DG}(\tau)}=\sum_{i=1}^{n_{\mathrm{Br}}} \sum_{j=1}^{n_{\mathrm{L}}} \frac{\left(V_{\mathrm{B}, \mathrm{DG}_{i, j}}^{(\tau)}\right)^{2}}{R_{i}} \Delta t_{j}
\end{aligned}
$$

The objective function related to the reliability of the EDS is proposed in (15). Reliability is measured by the costs of unserved energy by the EDS, considering three levels of demand, in response to actions of protective devices after the occurrence of transient and permanent faults, and these were restored by the DGs. In this context, it is assumed that during the interruption of power supply to a region served by the EDS, and being this region in isolated condition, the DGs could operate in islanded mode through an appropriate protection scheme, since the DGs are not connected to the contingency region. On the other hand, the costs of adapting the EDS refer to the acquisition, installation, and maintenance of protective devices for the purpose of operating the DGs in islanded mode, as proposed by the objective function (16). This expression consists of a fixed and a variable parts. The fixed part (PFC), given in (19), is directly related to the current injected by the DGs at the connection point of the EDS and is related to the purchase and installation of protection devices and to the replacement of keys that remain in trajectory between the SE and the DGs. The variable part is formed by the service cost (PSC), given in (20), and the maintenance cost (PMC), given in (21). The service cost comes from installing and uninstalling the protection devices, and the maintenance cost is the amount paid annually for the maintenance of the protection devices.

$$
\begin{aligned}
\operatorname{PFC}^{(\tau)} & =\sum_{i=1}^{n_{\mathrm{PD}}} \sum_{j=1}^{n_{\mathrm{In}}} \mathrm{AQC}_{i, j}^{(\tau)} \\
\operatorname{PSC}^{(\tau)} & =\sum_{i=1}^{n_{\mathrm{PD}}} \sum_{j=1}^{n_{\mathrm{In}}}\left(\operatorname{In}_{i, j}^{(\tau)}+\mathrm{Un}_{i, j}^{(\tau)}\right) \\
\operatorname{PMC}^{(\tau)} & =\sum_{i=1}^{n_{\mathrm{PD}}} \sum_{j=1}^{n_{\mathrm{In}}} \mathrm{MC}_{i, j}^{(\tau)} \varphi_{i}
\end{aligned}
$$

In the event of DGs operating in islanded mode, the mathematical formulation proposed in this section considers the installation of Overcurrent Relays with Directional and Synchronized Recloser Units (ORsD-SR). These devices are connected at the end bus of the selected branch for the installation of the DG and must be coordinated to operate in the downstream direction from the SS. In this study, the results obtained by Meneses (2012) regarding the protection devices allocation are used.

\section{Power Flow and Multi-Objective Optimization Algorithm}

In the proposed analysis, the power flow is an essential tool. Bearing in mind factors such as speed of convergence, accuracy, and robustness, a power flow based on the backward-forward method (Cheng and Shirmohammadi 1995 ) is used in this proposal.

The MO is based on the concept of Pareto optimality. A solution is said to be Pareto-optimal if not one of its objective functions can be improved without degrading all others, that 


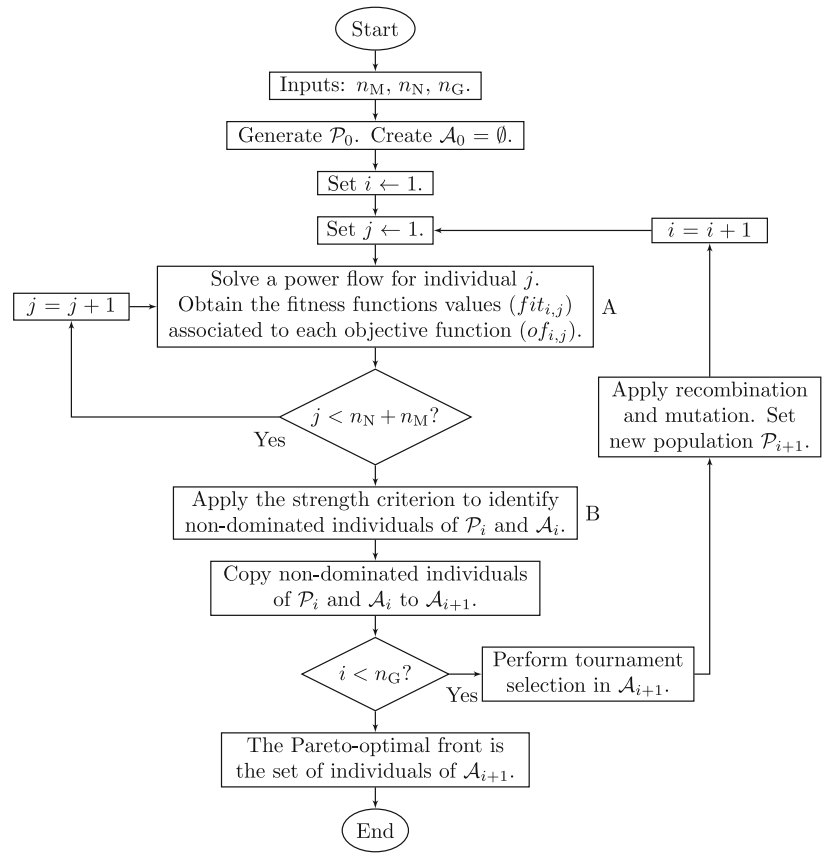

Fig. 1 Flowchart of the MO algorithm

is, a solution is Pareto-optimal if it is not "dominated" by others.

The problems formulated in this work are of a mixed integer nonlinear programing nature. Nonlinear constraints are handled by incorporating penalty functions directly to the MO algorithm. The proposed MO algorithm is based on an evolutionary optimization technique known as SPEA2 (Zitzler 1999). The objectives to be optimized through this algorithm, related to problems 1 and 2, were described in previous sections.

In a general view, for the solution of the proposed problems 1 and 2, the flowchart of the SPEA2 adapted to the MO algorithm is shown in Fig. 1. Note that, in Block A, a power flow must be run for each element of the sets $\mathcal{A}_{i}$ and $\mathcal{P}_{i}$ in order to obtain their fitness function (performance function) values associated with the objective functions of problems 1 and 2, which are computed according to (22) and (23). The strength criterion used in Block B is based on a mechanism that intuitively reflects the idea of preferring Pareto-optimal individuals and distributing them, at the same time, along a trade-off Pareto-optimal front (Zitzler 1999).

$$
\begin{aligned}
& f i t_{i, j}=o f_{i, j}+\beta_{i, j} \sum_{k=1}^{n_{\mathrm{V} i}} \Omega_{i, j, k} \\
& \Omega_{i, j, k}= \begin{cases}\omega_{i, j, k}\left(\underline{u}_{i, j, k}-u_{i, j, k}\right)^{2}, & \text { if } u_{i, j, k}<\underline{u}_{i, j, k} \\
\omega_{i, j, k}\left(u_{i, j, k}-\bar{u}_{i, j, k}\right)^{2}, & \text { if } u_{i, j, k}>\bar{u}_{i, j, k}\end{cases} \\
& \forall i=1,2, \ldots, n_{G}, \quad \forall j=1,2, \ldots, n_{M}+n_{N}
\end{aligned}
$$

The variables used in the codification to represent each individual of $\mathcal{A}_{i}$ and $\mathcal{P}_{i}$ are the active power generated by the DGs and the buses in which the DGs are proposed to be installed. These output variables feed the power flow, which delivers all the results necessary to compute the fitness functions values associated with the objective functions of problems 1 and 2 (22).

\section{Results and Discussion}

In this section, the results obtained for problems 1 and 2 from simulations performed in the 135-bus distribution test system, whose parameters are described in LaPSEE (2013), are presented and discussed. The computational implementations of the proposed methodologies for solving problems 1 and 2 were written in the Fortran programing language. The simulations were carried out on a computer with Intel Core i5 processor and $2000 \mathrm{MHz}$ of RAM.

The 135-bus distribution test system corresponds to a three-phase MV radial feeder, adapted from a city in the state of São Paulo, Brazil. The rated voltage of this test system is $13.8 \mathrm{kV}$ phase-phase. The feeder is composed by cables type ACSR 1/0, 2 and 4. In tests, a single-phase representation of the original system was used.

In this work, three load levels for the EDS were considered: light level with load factor of 0.25 and duration of $2555 \mathrm{~h} /$ year; middle level with load factor of 0.70 and duration of $4015 \mathrm{~h} /$ year; and nominal level with load factor of 1.00 and duration of $2190 \mathrm{~h} /$ year. Minimum and maximum voltage levels considered for the EDS were 0.95 and $1.05 \mathrm{pu}$, and the voltage at bus 1 (the SS) was fixed to 1.00 p.u.

In this work, it was decided to evaluate the proposed considering a single DG to be connected to the EDS with the exciter controlling the power factor, fixed at 0.92 in advance. The power ratings to be injected by the DG to the EDS were discretized in the range of minimum active power of $200 \mathrm{~kW}$ and a maximum active power of $1,800 \mathrm{~kW}$. An air interconnection line (from the DG to the EDS connection bus) which consists of a cable type ACSR 1/0 CAA with the cost of $9,763.71 \$ / \mathrm{km}$ was considered; this value is based on data obtained by consulting an electricity distribution company in the Midwest region of Brazil for the year 2013. A geographical location of the DG was supposed, and the distances to all buses of the EDS were determined. The considered costs of investment, operation and maintenance, and due to the use of the EDS, are 2,245.00 $\$ / \mathrm{kW}, 0.0055 \$ / \mathrm{kW}$ and $0.003 \$ / \mathrm{kW} / \mathrm{month}$, repectively.

\subsection{Results of Problem 1}

For problem 1, in order to perform comparative analysis, the valuation of energy losses (VEL), defined as one 
Table 1 Annual energy losses and associated costs without DGs installed in the EDS

\begin{tabular}{lcc}
\hline Load factor & Losses $(\mathrm{kWh}$ /year) & Losses cost $(\$)$ \\
\hline 0.25 (minimum) & $11,468.67$ & 584.90 \\
0.70 (medium) & $143,748.80$ & $11,931.15$ \\
0.25 (nominal) & $161,920.50$ & $16,192.05$ \\
Total & $317,137.97$ & $28,708.10$ \\
\hline
\end{tabular}

of the objective functions of problem 2, was computed (note that for problem 1 the objective function VEL is not part of the MO algorithm). For obtaining the VEL values, energy costs of $0.051,0.083$, and $0.100 \$ / \mathrm{kWh}$ for load factors of $0.25,0.70$, and 1.00 , respectively, were considered.

The simulation time is one year with an interest rate $(\alpha)$ of $11.31 \%$. The monetary values used in this work are proportional to those used in Pereira Junior (2014). It is also defined, as base case, the scenario in which there is no DGs installed in the EDS. In this condition, the annual energy losses and associated costs are presented in Table 1.

In Table 2, the active power generation of the DG delivered to the EDS, respective connection bus and section, section load, distance from the DG to the connection point in the EDS, associated allocation costs, and VEL values of each non-dominated solution obtained using the SPEA2 algorithm in problem 1 are presented. These results were obtained using as parameters of the SPEA2 algorithm a population and external sets with size 60 and 30, respectively, and a total number of generations of 200 .

In Table 2, note that all VEL values are positive. This means that in every possible connection of the DG scenario there is a reduction in energy losses, reflecting in attractive solutions to the EDS.

In Fig. 2, associated allocation costs and the VEL values for all non-dominated solutions of problem 1 are depicted.

In Table 2 and Fig. 2, note that, as the allocation costs increases, there is a tendency to increase the active power generation of the DG. It also must be noticed that, when the active power generation of the DG increases, the associated VEL value also increases. From the economic point of view, an increase in the VEL values is considered as a monetary gain (credit) for the DSO.

In order to making a decision by the IEP about which alternative is the most attractive, analysis from the two points of view (IEP and DSO) must be examined: (1) choose the alternative with the lower cost or (2) select the alternative with the largest active power generation. From a technical point of view, all solutions satisfy the constraints imposed by the mathematical model; therefore, the decision for the best solution is necessarily an economic evaluation of all alternatives.
To evaluate the solutions obtained using the SPEA2 and the interrelationship between the objective functions formulated for problem 1, solutions 1, 16, and 30 were marked in Table 2. In this sense, it is observed that the option with the smallest investment is solution 1 , with allocation costs of $\$$ $1,226,500.00$. This solution, however, proposes the lowest active power generation of the DG $(0.4738 \mathrm{MW})$. On the other hand, the option with the largest active power generation is solution 30 with $1.7427 \mathrm{MW}$ and allocation costs of $\$ 4,155,300.00$. Solutions 1 and 30 are the end points of the solutions presented in Table 2 and any other solution lies between these two points (for example, solution 16 with active generation of $1.0573 \mathrm{MW}$ and allocation costs of $\$$ 2,540,400.00).

Therefore, for the IEP, when considering economic aspects, the best alternative is the one that has the lowest allocation costs, say solution 1 . However, when considering operational aspects, such as the maximum active power generation of the DG, the best option is solution 30 . When comparing solutions 1 and 30, the power generation of the DG increases in $267.81 \%$, while the costs rise in $238.79 \%$, from $\$ 1,226,500.00$ to $\$ 4,155,300.00$. The decision of choosing one of these alternatives, or any other shown in Fig. 2 and Table 2, passes through the analysis of other aspects that are beyond the scope of this work such as, for example, the energy sales opportunity and the energy price for the DSO.

Another analysis scenario, from the point of view of the IEP, is the one that takes into account the benefits to the EDS. Considering Fig. 2, the solution with maximum VEL value (\$ 16,134.60 annually) is solution 30, and, as explained in previous paragraph, is the alternative with the most cost value for the IEP. Increasing the VEL values is, from the point of view of economic analysis, credit for the DSO because with the presence of the DG there is a reduction in losses and, hence, part of that energy lost without the presence of the GD returns (in credit) to the DSO. Therefore, considering these options, the IEP and the DSO would discuss the terms in which they would perform the electric power contracting, considering the market interests for both.

\subsection{Results of Problem 2}

In this section, the results obtained for the solution of problem 2 are presented. In order to implement the proposed model for the solution of this problem, the EDS was divided into sections, which are separated by protection devices, as shown in Fig. 3. These protection devices have the function of opening or closing to isolate these zones in the presence of contingencies.

In the proposal presented in this work, for the solution of problem 2, the results of problem 1 are used. In the proposed model for problem 2, the costs of protection devices (ORsD-SR), as well as costs associated with 
Table 2 Non-dominated solutions of problem 1

\begin{tabular}{|c|c|c|c|c|c|c|}
\hline Solution & $\begin{array}{l}\text { Active power } \\
\text { generation of } \\
\text { the DG (MW) }\end{array}$ & $\begin{array}{l}\text { Connection } \\
\text { bus/section }\end{array}$ & $\begin{array}{l}\text { Section load } \\
(\mathrm{kW})\end{array}$ & $\begin{array}{l}\text { Distance from } \\
\text { the DG to } \\
\text { the EDS }(\mathrm{km})\end{array}$ & $\begin{array}{l}\text { Investments } \\
\text { costs }\left(\times 10^{6} \$\right)\end{array}$ & $\operatorname{VEL}\left(\times 10^{3} \$\right)$ \\
\hline 1 & 0.4738 & $42 / 3$ & 223.4367 & 13.05 & 1.2265 & 4.4803 \\
\hline 2 & 0.5497 & $88 / 18$ & 54.1267 & 8.03 & 1.3535 & 6.0114 \\
\hline 3 & 0.6661 & $87 / 18$ & 54.1267 & 8.51 & 1.6282 & 7.1668 \\
\hline 4 & 0.6792 & $7 / 2$ & 174.8000 & 12.16 & 1.6457 & 7.0889 \\
\hline 5 & 0.7102 & $97 / 9$ & 213.4100 & 11.65 & 1.7610 & 7.3681 \\
\hline 6 & 0.7409 & $15 / 3$ & 223.4367 & 11.90 & 1.8346 & 6.5915 \\
\hline 7 & 0.7546 & $84 / 9$ & 213.4100 & 10.73 & 1.8639 & 7.7681 \\
\hline 8 & 0.7770 & $14 / 2$ & 174.8000 & 11.06 & 1.8693 & 7.8813 \\
\hline 9 & 0.7780 & $92 / 12$ & 103.3467 & 10.34 & 1.9054 & 8.4227 \\
\hline 10 & 0.7922 & $12 / 2$ & 174.8000 & 11.18 & 1.9467 & 7.0280 \\
\hline 11 & 0.7954 & $44 / 4$ & 244.7200 & 10.71 & 1.9495 & 7.9879 \\
\hline 12 & 0.8117 & $27 / 5$ & 76.6367 & 10.71 & 1.9856 & 8.7564 \\
\hline 13 & 0.8306 & $54 / 8$ & 119.6000 & 8.65 & 2.0111 & 8.3348 \\
\hline 14 & 0.8315 & $64 / 10$ & 59.3100 & 8.96 & 2.0162 & 8.6148 \\
\hline 15 & 0.8360 & $47 / 5$ & 76.6367 & 9.42 & 2.0310 & 8.4212 \\
\hline 16 & 1.0573 & $50 / 8$ & 119.6000 & 9.02 & 2.5404 & 10.3014 \\
\hline 17 & 1.0669 & $77 / 11$ & 243.8000 & 9.58 & 2.5681 & 10.8280 \\
\hline 18 & 1.0779 & $91 / 11$ & 243.8000 & 10.69 & 2.5937 & 10.9216 \\
\hline 19 & 1.1302 & $55 / 5$ & 76.6367 & 9.16 & 2.7109 & 11.0664 \\
\hline 20 & 1.1367 & $59 / 5$ & 76.6367 & 9.14 & 2.7259 & 11.2470 \\
\hline 21 & 1.5242 & $70 / 9$ & 213.4100 & 9.85 & 3.6896 & 11.4033 \\
\hline 22 & 1.1795 & $30 / 4$ & 244.7200 & 10.03 & 2.8338 & 10.1945 \\
\hline 23 & 1.2144 & $34 / 3$ & 223.4367 & 12.90 & 2.9307 & 12.2891 \\
\hline 24 & 1.2569 & $7 / 2$ & 174.8000 & 12.16 & 2.9858 & 12.4786 \\
\hline 25 & 1.3111 & $3 / 2$ & 174.8000 & 13.05 & 3.1153 & 12.9705 \\
\hline 26 & 1.4036 & $24 / 3$ & 223.4367 & 12.30 & 3.3758 & 10.6885 \\
\hline 27 & 1.4283 & $21 / 3$ & 223.4367 & 12.18 & 3.4318 & 11.4030 \\
\hline 28 & 1.1612 & $8 / 2$ & 174.8000 & 11.91 & 3.6516 & 11.7223 \\
\hline 29 & 1.5427 & $56 / 7$ & 92.0000 & 10.43 & 3.6801 & 13.7778 \\
\hline 30 & 1.7427 & $37 / 2$ & 174.8000 & 10.26 & 4.1553 & 16.1340 \\
\hline
\end{tabular}

installation/uninstallation and maintenance of such equipment devices, are considered. The costs presented in tables 3 and 4, from Meneses (2012), as well as data obtained by consulting an electricity distribution company in the Midwest region of Brazil for the year 2013, were used in simulations.

For problem 2, in order to perform analysis, energy costs of $0.061,0.097$, and $0.120 \$ / \mathrm{kWh}$ for load factors of 0.25 , 0.70 , and 1.00 , respectively, were considered for obtaining the VEL values.

As for problem 1, the simulation time is one year with an interest rate $(\alpha)$ of $11.31 \%$, which is proportional to values used in Pereira Junior (2014).

According to the methodology proposed in this study to solve problem 2, through the SPEA2 and considering 30 scenarios for the connection of the DG, detailed results, such as active power generation of the DG, respective connection and section bus, VEL, VNE and SAC values, and IEP's investments costs (from problem 1) are presented in Table 5.

Annual energy losses without DG, which represent an additional cost related to the energy supplied to consumers, are \$28,708.10. Based on the results presented in Table 5, note that solution 1 has the smallest value for VEL. This is due to the fact that the annual energy losses in the EDS with DG are \$24.229,80 in this scenario, and the difference between these values (without and with DG) is $\$ 4,803.00$, which means a reduction in energy losses.

Considering solution 1 , note that the reliability cost is $\$ 42,779.30$, corresponding to the value of annual energy not supplied by the EDS, which was restored by the DG. According to the methodology adopted in this work, in the 


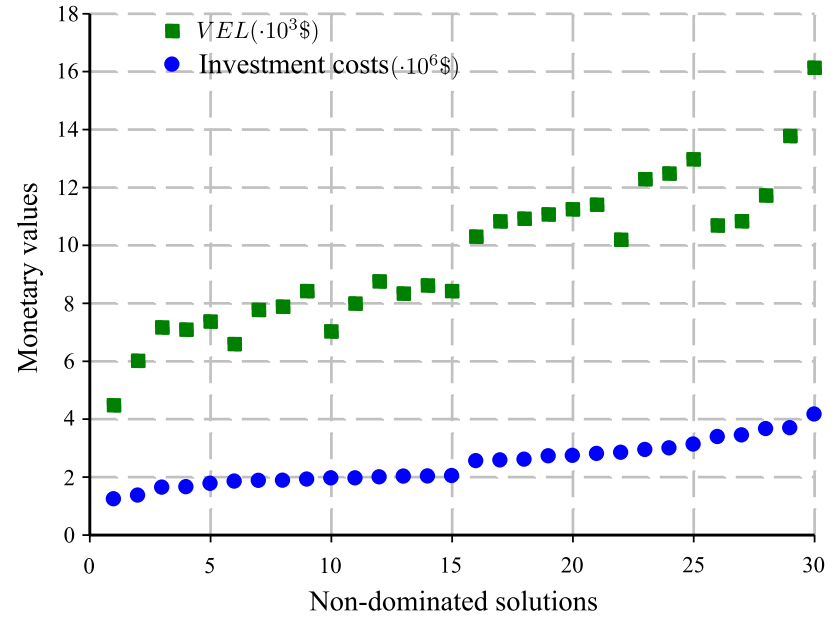

Fig. 2 Associated allocation costs and VEL values in problem 1

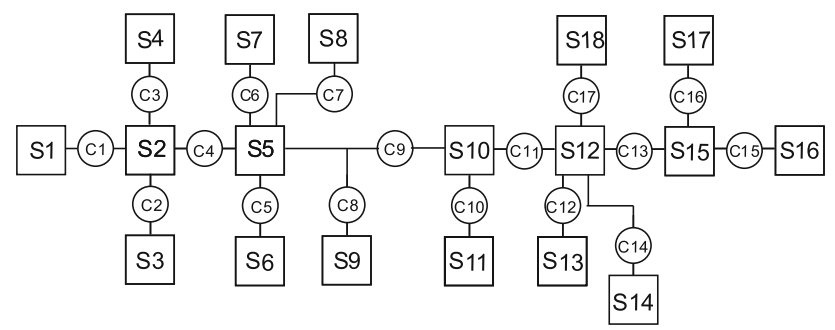

Fig. 3 135-bus distribution test system divided in sections ( $S$ corresponds to Section and $C$ corresponds to protection device)

definition of the sections, bus 42 belongs to Section 3, with load of $223.4367 \mathrm{~kW}$. Thus, the amount of $\$ 42,779.30$ is interpreted as a reduction in costs and it was obtained by considering the occurrence of contingency at some point of the EDS, out of Section 3, and being the DG connected to this section operating in islanded mode, providing energy to consumers and increasing the reliability of the EDS. This also causes a decrease in the number of consumers who are without energy, avoiding the payment of penalties. Therefore, this represents a benefit of the connection of the DG to the EDS which could be taken into account in decision-making about the bus in which the DG will be connected.

In Table 5, SAC has the value of $\$ 10,071.80$ for solution 1 , which corresponds to the costs of installation of an ORsD-SR. This relay, which should be installed in the line connecting buses 11 and 10 , should replace key 2 , which is between the SS and the DG. These protection devices allow the DG operates in islanded mode after a contingency somewhere in the system, except in the section in which the DG is connected to.

Comparing the values presented in Table 5 for solutions 1 and 2, note that solution 2, which connects the DG in bus 97 located in Section 9 with load of $213.41 \mathrm{~kW}$, has a VEL value greater than solution 1 . This occurs due to the fact that the active power generated by the DG in solution 2 is 0.7102
Table 3 Costs of protection devices

\begin{tabular}{lll}
\hline Device & Current inverval (A) & Cost (\$) \\
\hline Overcurrent relay & $0-50$ & 4000 \\
& $51-100$ & 4500 \\
$101-200$ & 5000 \\
$201-500$ & 5500 \\
$501-1000$ & 6000 \\
ORD-SR & $0-50$ & 6000 \\
& $51-100$ & 6500 \\
$101-200$ & 7000 \\
& $201-500$ & 7500 \\
& $501-1000$ & 8000 \\
\hline
\end{tabular}

Table 4 Costs associated with installation/uninstallation and maintenance of protection devices

\begin{tabular}{llll}
\hline Associated cost & Overcurrent relay & ORD-SR & Fuse link \\
\hline Installation/uninstallation & 1000 & 1500 & 100 \\
Maintenance & 500 & 750 & 50 \\
\hline
\end{tabular}

MW, causing lower losses in the EDS. As revealed in this study, the VEL value increases, causing greater benefits for the EDS.

As shown in Table 5, as the active power generation of the DG increases, there is also an increase in the SAC values. This is since in the model implemented for problem 2 the protection devices costs are defined in terms of the electrical current injected by the DG in the EDS, which depends on the active power to be generated by the DG. From this point of view, and considering the solutions shown in Table 5, the electrical current injected by the DG is in the range of 19.82 A to $72.91 \mathrm{~A}$ from the scenario of lower active power generation to the scenario of higher active power generation, respectively. As presented in Table 3, the overcurrent relays that operate in the range up to $50 \mathrm{~A}$ have cost of $\$ 4,000.00$, while overcurrent relays that operate in the range of $51 \mathrm{~A}$ to 100 A have cost of $\$ 4,500.00$. The ORsD-SR devices with the possibility of adjustment in the range up to $50 \mathrm{~A}$ and $51 \mathrm{~A}$ to 100 A have cost of $\$ 6,000.00$ and $\$ 6,500.00$, respectively. Thus, in the range of nominal current for the scenarios with the DG, costs of protection devices, which enables the DG operates in islanded mode, have a variation in the order of $60.19 \%$.

In Table 5, it is shown that the reliability cost for all alternatives has a behavior almost constant. These costs represent the energy provided by the DG when operating in islanded mode in the presence of any contingency in the EDS. As can be observed in this table, the non-dominated solutions for problem 2 define sections 3, 9, 4, and 11 as the connection point of the DG. In Table 2, it can be observed that sections 
Table 5 Non-dominated solutions of problem 2 and IEP's investments costs (from problem 1)

\begin{tabular}{lllllll}
\hline Solution & $\begin{array}{l}\text { Active power } \\
\text { generation of the } \\
\text { DG }(\mathrm{MW})\end{array}$ & Connection bus & VEL $\left(\times 10^{3} \$\right)$ & VNE $\left(\times 10^{3} \$\right)$ & SAC $\left(\times 10^{3} \$\right)$ & $\begin{array}{l}\text { PIE's Investments costs } \\
\left(\times 10^{6} \$\right)\end{array}$ \\
\hline 1 & 0.4738 & 42 & 4.4803 & 42.7793 & 10.0718 & 1.2265 \\
2 & 0.7102 & 97 & 7.3681 & 40.8597 & 12.1278 & 1.7610 \\
3 & 0.7546 & 84 & 7.7681 & 40.8597 & 12.5139 & 1.8639 \\
4 & 0.7954 & 44 & 7.9879 & 46.8542 & 12.8691 & 1.9495 \\
5 & 1.0669 & 77 & 10.8280 & 46.6782 & 15.2307 & 2.5681 \\
6 & 1.0779 & 91 & 10.9216 & 46.6782 & 15.3268 & 2.5937 \\
7 & 1.4283 & 21 & 11.4030 & 42.7793 & 18.3747 & 3.4318 \\
8 & 1.5242 & 70 & 11.4033 & 40.8597 & 16.0514 & 3.6896 \\
9 & 1.7427 & 37 & 11.4342 & 46.8542 & 16.1340 & 4.1553 \\
\hline
\end{tabular}

$3,9,4$, and 11 are those with the highest installed loads and with values very close. This explains the fact that the reliability costs are almost constant in scenarios for problem 2 . In turn, sections 3, 4, 9, and 11, are for the DSO the best connection alternatives of the DG reducing losses for energy not supplied.

Evaluating all the alternatives presented in Table 5, note that the best set of alternatives for the DSO is solutions 4, 5,6 , and 9. These solutions connect the DG in buses 44,77 , 91 , and 37 , respectively, and these buses belong to sections 4 and 11.

Based on the obtained results, it can be said that the best solutions for the DSO are solution 4 or 9 , in which the DG is connected to bus 44 with active power of $0.7954 \mathrm{MW}$, and to bus 37 with active power of $1.7427 \mathrm{MW}$, respectively. Solution 4 causes a decrease in the annual energy losses of the EDS in $5.10 \%$, while solution 9 decreases the annual energy losses in $7.30 \%$. In these scenarios, the annual reliability cost has a value in the order of $\$ 46,854.20$, which represents a payback for the DSO because of the DG be operating continuously and providing active power in the event of contingencies in the EDS. Operating the DG in islanded mode causes investments cost of $\$ 12,869.10$ in solution 4 , $\$ 15,230.70$ in solution $5, \$ 15,326.80$ in solution 6 and $\$$ $16,134.40$ in solution 9. Comparing these values, the DSO makes an initial investment and guarantees the return on this investment in the short term.

In Table 5, a comparison of the annual cost for the IEP and the DSO for the scenarios of allocation of the DG that reflect interests for both are presented. In this table, it is observed that the most attractive scenario for IEP, from the point of view of amount of required investment, is solution 1; however, this is not the most attractive alternative if the interests of DSO are considered. For the DSO, the most attractive alternatives are solutions 4 and 9. For the formulated test case, just the fact of connecting the DG brings benefits to the DSO, given that this connection increases the VEL value.
Another beneficial effect observed is related to the reliability of the EDS. The amount of investment required from the DSO in protection devices that will allow the DG operates in islanded mode, if compared to the reliability cost that is the monetary value that the DSO will receive in a year, allows defining solutions 4 and 9 as the most attractive for the DSO. However, for the IEP, evaluating from an economic point of view, solution 9 is the worst solution, because it requires a high investment.

In Table 5, it is also observed that when the DG has the lowest generation capacity, the amount of investment required from the IEP (\$1.226.500,00), as well as from the DSO (\$ 10,071.80), is the smallest monetary value. When the extent to which the capacity of the DG increases, there is an increase in investments for both, the IEP and the DSO; however, this increase is more significant for the IEP.

The best solutions for the IEP and one of the best solutions for the DSO are the extremes of Table 5. Solution 1 is applicable for the IEP when considering the hypothesis of minimum overall cost. For the DSO, solution 9 is applicable when considering their interests, because in this solution the valuation of energy losses is maximized and the cost of reliability is minimized. Thus, these extreme solutions are conflicting in the discussion of a contract of purchase and sale of energy between the IEP and the DSO. In this sense, the other solutions could be analyzed for choosing the one that is more attractive to the IEP and to the DSO, or the one that provides lower costs to the consumers.

\section{Conclusions}

In this work, a study of technical and economic evaluation for the allocation of DGs in EDSs from the perspective of the IEPs and the DSO is conducted. There were two MO problems defined named as problem 1 , from the IEPs's per- 
spective, and problem 2, from the DSO's perspective. These problems were solved by using the MO algorithm called SPEA2. The proposal was implemented using data from the 135-bus distribution test system and assuming a single DG to be connected to the EDS.

According to this study, for problem 1 , any of the solution alternatives could be adopted by the IEP. As can be noticed in these results, with the lowest generation capacity of the DG, the costs have the lowest value; meanwhile, with the larger generation capacity of the DG, the costs reach the highest value.

The problem 2 was solved considering as search space the non-dominated solutions found when solving problem 1. To solve problem 2, a MO algorithm was implemented to find the non-dominated solutions that represent the interest of the DSO. Among the solutions of problem 1, the solution algorithm of problem 2 returned a new set of non-dominated solutions representing the DG interconnection alternatives which meet the expectations of the DSO, presenting the investment required to adapt the EDS, as well as credit values for the DSO. Then, with these results, the DSO can evaluate the financial return that each solution promotes and decides the best connection alternative for the DG.

Comparing the costs for the IEP and the DSO and considering that at this stage the set of solutions for the connection of the DG comes down from the solutions found when solving problem 1, it is observed that one of the most attractive options for the DSO is the less attractive alternative for the IEP, from the economic point of view. As it can be noticed, some solutions result in savings for the DSO and low investment for the IEP. According to this study, any of these solutions could be adopted to establish a negotiation between the IEP and the DSO because they meet the expectations of both. It could also be found the solution that brings the lowest overall cost, which may be the most interesting for the consumers.

\section{References}

Abou El-Ela, A. A., Allam, S. M., \& Shatla, M. M. (2010). Maximal optimal benefits of distributed generation using genetic algorithms. Elsevier Electric Power Systems Research, 80(7), 869-877.

Agência Nacional de Energia Elétrica (ANEEL). (2005). Procedimentos de distribuição de energia elétrica no sistema elétrico nacional (PRODIST): Módulo 2: Planejamento da expansão do sistema de distribuição. On line: www.aneel.gov.br/arquivos/PDF/Modulo2_ Revisao_4- Final (in portuguese).

Atwa, Y. M., \& El-Saadany, E. F. (2011). Probabilistic approach for optimal allocation of wind-based distributed generation in distribution systems. IET Renewable Power Generation, 5(1), 79-88.

Barker, P. P. \& Demello, R. W. (2000). Determining the impact of distributed generation on power systems: Part 1- radial distribution systems. In IEEE power engineering society summer meeting, 2000 (pp. 1645-1656).

Brown, R. E. \& Freeman, L. A. A. (2001). Analyzing the reliability impact of distributed generation. In IEEE power engineering society summer meeting, 2001 (pp. 1013-1018).
Cheng, C. S., \& Shirmohammadi, D. (1995). A three-phase power flow method for real-time distribution system analysis. IEEE Transactions on Power Systems, 10(2), 671-769.

Ganguly, S., \& Samajpati, D. (2015). Distributed generation allocation on radial distribution networks under uncertainties of load and generation using genetic algorithm. IEEE Transactions on Sustainable Energy, 6(3), 688-697.

Ghosh, S., Ghoshal, S. P., \& Ghosh, S. (2010). Optimal sizing and placement of distributed generation in a network system. Elsevier International Journal of Electrical Power \& Energy Systems, $32(8), 849-856$.

Gözel, T., \& Hocauglu, M. H. (2009). An analytical method for the sizing and siting of distributed generators in radial systems. Elsevier Electric Power Systems Research, 79(6), 912-918.

Gupta, P., Pandit, M. \& Kothari, D. P. (2014). A review on optimal sizing and siting of distributed generation system: Integrating distributed generation into the grid, IEEE power India international conference, 2014 (pp. 1-6).

Hsieh, S (2011). Bi-Objective planning for distributed generations in distribution systems using particle swarm optimization and compromise programming. In Conference on electric utility deregulation and restructuring and power technologies (DRPT), 2011 (pp. 168-173).

Keane, A., \& O'Malley, M. (2005). Optimal allocation of embedded generation on distribution networks. IEEE Transactions on Power Systems, 20(3), 1640-1646.

Laboratório de Planejamento de Sistemas de Energia Elétrica (LaPSEE). (2013). On line: www.dee.feis.unesp.br/lapsee/, Faculdade de Engenharia Elétrica de Ilha Solteira, Universidade Estadual Paulista-UNESP (in portuguese).

Mahmoud, K., Yorino, N., \& Ahmed, A. (2015). Optimal distributed generation allocation in distribution systems for loss minimization. In IEEE Transactions on Power Systems, 99, 1-10.

Meneses, C. A. P. (2012). Desenvolvimento de técnicas e modelos matemáticos para solução de problema de planejamento da expansão e operação de sistemas de distribuição de energia elétrica com geradores distribuídos, PhD thesis, Faculdade de Engenharia Elétrica de Ilha Solteira, Universidade Estadual Paulista_-UNESP (in portuguese).

Nazari, H. N. \& Parniani, M. (2006). Determining and optimizing power loss reduction in distribution feeders due to distributed generation. In Power systems conference and exposition, 2006 (pp.1914-1918).

Neimane, V. (2001). On development planning of electricity distribution networks, PhD thesis, Department of Electrical EngineeringRoyal Institute of Technology, Stockholm.

Nuroglu, F. M. \& Arsoy, A. B. (2008). Voltage profile and shortcircuit analysis in distribution systems with DG. In Electric power conference, 2008 (pp. 1-5).

Ochoa, L. F., Padilha-Feltrin, A., \& Harrison, G. P. (2008). Evaluating distributed time-varying generation through a multiobjective index. IEEE Transactions on Power Delivery, 23(2), 1132-1138.

Pereira Jr, B. R. (2014). Planejamento de médio e longo prazo de sistemas de distribuição de energia elétrica com geradores distribuídos considerando cusos de confiabilidade, operação e expansão, $\mathrm{PhD}$ thesis, Faculdade de Engenharia Elétrica de Ilha Solteira, Universidade Estadual Paulista-UNESP (in portuguese).

Rau, N. S., \& Wan, Y. H. (1994). Optimum location of resources in distributed planning. IEEE Transactions on Power Systems, 9(4), 2014-2020.

Wen, S., Lan, H., Fu, Q., Yu, D. C., \& Zhang, L. (2015). Economic allocation for energy storage system considering wind power distribution. IEEE Transactions on Power Systems, 30(2), 644-652.

Zitzler, E. (1999). Evolutionary algorithms for multiobjective optimization: Methods and applications, $\mathrm{PhD}$ thesis, Swiss Federal Institute of Technology. 